\title{
Biology and Genetics of Human Longetivity
}

\author{
Giovannella Baggio \\ University of Sassari, Italy \\ Claudio Franceschi \\ University of Modena and INRCA, Italy \\ Daniela Mari \\ University of Milan and IRCCS Maggiore Hospital, Italy \\ A.M. Herskind, Karen Andersen-Ranberg, Bernard Jeune \\ Odense University, Denmark
}

\begin{abstract}
Human longevity is a multifactorial trait, which includes a network of genes combined with strong environmental factors. Biological and clinical characteristics of human beings are the result of the interaction between genes and the environment. Until about 1950 centenarians were quite rare in low-mortality countries. Since then the number has more than doubled every 10 years and even the number of 105+ year old people is now increasing. This proliferation of centenarians is mainly a result of the decrease in oldest-old mortality and probably due to changes of lifestyle and health care. Although studies of Danish twin pairs seem to indicate that genetic influence on human lifespan is only moderate, several gene loci contribute to longevity. Data are evident from the Italian Centenarian Study for apoproteinB, tyrosine hydroxylase and mitochondrial DNA loci among others studied (superoxide dismutases, ie. SOD1 and SOD2, poly(ADP-ribose)polymerase and thyroid peroxidase). The data from the Danish Centenarian Study confirm the findings from the studies of French and Finnish centenarians on apolipoprotein $\mathrm{E}$ genotype with a higher frequency of epsilon-2 and lower frequency of epsilon4. However, the apoE genotype seems not to be a key determinant of exceptional longevity: in $105+$ year olds the epsilon- 4 allele was found in 4 out of 19 persons. Italian centanarians have a paradoxically marked hypercoagulability as demonstrated also by genetic markers. Also the von Willebrand factor was increased independently of the blood group. Studies on such atherosclerosis risk factors as lipoprotein(a) and homocysteine revealed that these two parameters may be high in Italian centenarians, but their genetic control possibly attenuates with age, and environmental factors may play a major role in the oldest-old persons. Also interaction among genes is possible. All these studies suggest that longevity is a phoenomenon depending on multiple genetic and environmental factors. Further studies are needed for a better understanding of the complex interactions which allow people to reach a very old age.
\end{abstract}

\section{The Proliferation of Centenarians}

Until about 1950 centenarians were quite rare. The prevalence of centenarians in Denmark was only 1-2 per million in the last half of the 1800s [1]. The number of reported centenarians before 1850 was most plausibly due to age-exaggeration. It is possible that there were no true centenarians prior to 1800 [2]. In the first half of the $1900 \mathrm{~s}$ their numbers grew only slowly. Then for cohorts born after 1860 the probability of becoming a centenarian increased substantially. Since 1950 their numbers have more than doubled every 10 years in lowmortality countries, resulting in a prevalence of about 80 per million in 1995 in Denmark [3].

The main proportion of the rate of growth is due to a decrease in oldest-old mortality. Today centenarians also live longer, approximately two years on average. As a result, the number of centenarians reaching age 105 in the Nordic countries has increased since 1970. Mainly nongenetic and probably period effects, like changes in lifestyle and health care, have therefore created a

Correspondence to Giovannella Baggio, Chair of Geriatrics and Gerontology, University of Sassari, Viale San Pietro 8,07100-Sassari, Italy. Email: baggio@ssmain.uniss.it new generation. The average life span has been increasing as a result of non-genetic factors, but variation in life span within a cohort is influenced by genetics factors. Danish twin studies have estimated the heritability of human life span as approximately. $25 \%$.

\section{The Heritabilty of Lifespan}

A study was performed in order to explore the nature (ie. additive versus non-additive) and magnitude (ie. heritability) of genetic influences on inter-individual variation in human lifespan and, furthermore, to evaluate how these genetic influences on lifespan are mediated, specifically to what extent they are mediated through genetic influences on smoking behavior and obesity [4]. Both of these factors have a substantial genetic component and in addition both have a major influence on mortality.

The studies were based on two different subsamples of the Danish Twin Register [5]. The univariate study of lifespan comprised all like-sexed twin pairs born in Denmark in the period 1870-1900, yielding a total of 2,872 pairs. By 1994 all but $0.6 \%$ had died, making the sample almost non-censored. The multivariate study was based on a 28-year 
follow-up of a sample of 1,232 Danish twin pairs born 1890-1920 who answered a questionnaire in 1966. Proportions of covariance due to genetic and environmental factors in common and unique to the three traits were estimated from variancecovariance matrices using the structural equation model approach. Genetic influences on human lifespan were only moderate. The heritability was estimated to be 0.25 , slightly higher for males than for females, but constant over birth-cohorts, with genetic factors being non-additive due to gene interaction within loci. Only a small and nonsignificant fraction (5-9\%) of the genetic influences on lifespan was mediated through genetic influences on smoking and obesity.

\section{The Genetics of Longevity}

Some possible candidate genes. Other studies of Italian centenarians suggested that human longevity is a multifactorial trait [6]. The hypothesis is that several genes acting as part of a network interact with a strong environmental influence. Therefore their individual contribution is expected to be low. We reasoned that candidate genes for longevity should be involved in pathways responsible for soma integrity, such as the network of cellular defence mechanisms whose efficiency is crucial to repair the continuous damages due to internal and internal agents (genes involved in free radical homeostasis, heat shock proteins, DNA repair, apoptosis) or should play important roles in metabolic pathways (lipid homeostasis, neurotransmitter synthesis).

To test this hypothesis, 200 centenarians and 300 younger people, ranging from 20 to 60 years, were genotyped for polymorphisms located within or close to the following genes: superoxide dismutases (SOD1 and SOD2), mitochondrial DNA haplotypes (mtDNA), poly(ADP-ribose)polymerase (PARP), apolipoprotein B (ApoB) [7], tyrosine hydroxylase (TH) and thyroid peroxydase (TPO). Allelic and genotypic frequency distributions were analysed. ApoB, TH, mtDNA loci showed significant differences when centenarians and younger people were compared. Differences between centenarians of northern and southern Italy were also found at the TH locus.

Our data are in line with the assumption that several loci contribute to longevity. In addition, given that our analysis is performed on the same group of subjects, we expect to obtain data on possible interactions among these genes.

Apolipoprotein $\mathrm{E}$ gene and exceptional longevity. Previous studies of French and Finnish centenarians indicate differential mortality among apolipoprotein $\mathrm{E}$ (apoE) genotypes at older ages [8]. We have determined the apoE genotypes in 146 out of 207 Danes who were examined just after their 100 year birthday, and in 19 out of the 24 Danes who were 105 years old or older. They were compared with the frequency in a sample of 466 Danes aged 40. The data support the findings from the French and the Finnish centenarians showing a higher frequency of the epsilon-2 allele among Danish centenarians (11.3\% at age 100 and $10.5 \%$ at age
$105+$ vs. $8.5 \%$ at age 40 ) and a much lower frequency of the epsilon- 4 allele ( $7.2 \%$ at age 100 and $10.5 \%$ at age $105+$ vs. $17.4 \%$ at age 40 ). However, the data do not support the hypothesis that apoE genotype is a key determinant of exceptional longevity.

As only about $5 \%$ of 100 year old Danes survive five years, the frequency of the epsilon-4 allele could be expected to be even lower and the epsilon-2 allele even higher in Danes older than 100 and in those extraordinary people older than 105 years. In fact the opposite was observed and 4 of the 105+ year olds carried the epsilon- 4 allele. Although there were no epsilon-4/epsilon-4 genotypes among the 105+ year old Danes, one 100 year old Dane carried the epsilon/epsilon-4 genotypes.

\section{Some Biological Markers and Paradoxes}

Studies on haemostasis. Subjects with blood type $O$ have lower concentration of von Willebrand factor (vWF) than those with blood group non-O. Since we recently observed that laboratory signs of marked hypercoagulability and some corresponding genetic marker are compatible with health and longevity in Italian centenarians [9-11], we determined $\mathrm{vWF}: \mathrm{Ag}$ and blood groups to see if levels of this marker of endothelial damage were altered. We also performed multimeric analysis of vWF. We studied 74 (age range 100 to $107 \mathrm{yrs} ; 18 \mathrm{M}$ and $56 \mathrm{~F}$ ) and 110 controls (aged from 21 to $86 \mathrm{yrs} ; 49 \mathrm{M}$ and $61 \mathrm{~F}$ ).

The levels of $\mathrm{vWF}: \mathrm{Ag}$ in centenarians were significantly higher than in the controls. No significant difference was observed between vWF:Ag levels in centenarians with blood group $\mathrm{O}$ or non-O: 245 (215-279) vs. 285 (251-325) U/dL. The controls with blood group $\mathrm{O}$ have lower levels of $\mathrm{vWF}: \mathrm{Ag}$ than those with blood group non-O: younger controls 77 (67-91) vs. $115(100-132) \mathrm{U} / \mathrm{dL} \mathrm{p}=0.0006$; older controls $99(84-117)$ vs. $152(133-174) \mathrm{U} / \mathrm{dL} \mathrm{p}=$ 0.0001 . In half of the centenarians there was a reduction in the relative proportion of high molecular weight multimers, calculated by scanning the autoradiographs with a densitometer. Thus, vWF, an independent predictor of atherothrombotic disease, was increased in centenarians, independently of the blood group, confirming the previous results of a paradoxical state of hypercoagulability in healthy centenarians.

Studies on lipoproteins and proinflammatory cytokines. $\mathrm{Lp}(\mathrm{a})$ levels, lipoprotein profile, and proinflammatory cytokines (IL-6, TNF-alfa) were measured in 75 centenarians and in control subjects of different ages [12]. The most unexpected findings were:

- $25 \%$ of healthy centenarians had $\mathrm{Lp}(\mathrm{a})>30$ $\mathrm{mg} / \mathrm{dl}$;

- only the centenarians with high $\mathrm{Lp}$ (a) levels showed increased values of proinflammatory cytokine IL- 6;

- total and LDL cholesterol levels in centenarians were similar to young controls, but significantly lower than in elderly controls, while $\mathrm{HDL}$ cholesterol was lower and triglycerides were higher than in young subjects. 
We suggest that genetic control of $\mathrm{Lp}(\mathrm{a})$ may attenuate with age and that environmental factors (chronic subclinical inflammatory processes?) may play a major role in determining the blood levels of $\mathrm{Lp}(\mathrm{a})$ in the oldest old.

Study on homocysteine. Homocysteine, vitamin B12 and folic acid levels were determined in 47 centenarians. Mean homocysteine levels were $23.5 \pm$ 14.7 micron $\mathrm{mol} / \mathrm{L}$ vs. $14.6 \pm 3.5$ in elderly controls, medium grade hyperhomocysteinemia ( $>30$ micron moll) was observed in 10 subjects $(21.2 \%)$; two of these presented macrocytic anemia with low vitamin B12 values, while two showed severe folate deficiency. Homocysteine values were significantly correlated with folates $(r=-0.395, p=0.027)$ but not with vitamin $\mathrm{B} 12$, while the significant correlation with creatinine observed in other case reports was confirmed ( $r=0.383, p=0.008$ ). These results suggest that mild hyperhomocysteinemia in very old people may be linked to nutritional deficiencies present in later life, so the impact of this factor on vascular risk may be less pronounced than in younger individuals.

\section{References}

1. Jeune B, Skytthe A, Vaupel JW. Demography of centenarians in Denmark. Ugeskr Leeger 1996;158:7392-6.

2. Jeune B, Vaupel JW, Eds. Exceptional longevity: from prehistory to the present. Monographs on Population Aging, vol. 2. Odense, Denmark: Odense University Press, 1995.

3. Jeune B, Kannisto V. Emergence of centenarians and supercentenarians. In: Robine J-M, Vaupel JW, Jeune B, Allard $M$, eds. Longevity: to the limits and beyond. Berlin, Heidelberg: Fondation Ipsen, Springer Verlag, 1997.

4. Herskind AM, McGue M, Yashin I, Holm NV, Sorensen TIA, Harvald B, Vaupel JV. Untangling the genetic influences on smoking, body mass index and longevity: a multivariate study of 2464 Danish twins followed for 28 years. Human Genetics 1996;98:467- 75.
5. Herskind AM, McGue M, Holm NV, Sorensen TIA, Harvald B, Vaupel JV. The heritability of human longevity: a population based study of 2,872 Danish twin pairs born 1870-1900. Human Genetics 1996;97:319-23.

6. De Benedictis G. Genes and longevity. Aging: Clinical and Experimental Research 1996; 8:367-369.

7. De Benedictis G, Falcone $E$, Rose G, Ruffolo $R$, Spadafora P, Baggio G, Bertolini S, Mari D, Mattace R, Monti D, Morellini M, Sansoni P, Franceschi C. DNA multiallelic systems reveal gene-longevity associations not detected by diallelic systems: the apo-B locus. Human Genetics 1997; 99:312-318.

8. Schachter F, Faure-Delanef $L$, Guenot $F$, Rouger $H_{\text {, }}$ Froguel $P$, Lesueur-Ginot L, Cohen D. Genetic association with human longevity at the apoE and $A C E$ loci. Nature Genet 1994; 6:29-32.

9. Mari D. Mannucci PM, Coppola R, Bottasso B, Bauer KA, Rosenberg RD. Hypercoagulability in centenarians: the paradox of successful aging. Blood 1995;85: 3144-3149.

10. Mari D, Mannucci PM, Duca F, Bertolini S, Franceschi C. Mutant factor $V$ (Arg506Gin) in healthy centenarians. Lancet 1996;347:1044.

11. Mannucci PM, Mari D, Merati G, Tagliabue L, Sacchi E, Tajoli F, Bertolini S, Franceschi C. Gene polymorphisms predicting high plasma levels of coagulation and fibrinolysis proteins: a study in centenarians. Arterioscler Throm Vasc Biol 1997;17: 755-759.

12. Baggio G, Donazzan S, Monti D, Mari D, Martini S, Gabelli C, Dalla Vestra M, Previato L, Guido M, Pigozzo S, Cortella I, Crepaldi G, Franceschi C: Lipoprotein(a) and lipoprotein profile in healthy centenarians: a reappraisal of vascular risk factors. FASEB Journal 1998; 12: ( in press).
Giovannella Baggio convened the Symposium on Biology and Genetics of Human Longevity, World Congress of Gerontology, Adelaide, Australia, 1997. It was co-chaired by Claudio Franceschi. The following papers were presented:

Andersen-Ranberg $K$. Is the apoliprotein $E$ gene a determinant of exceptional longevity?

Baggio G. Biology and genetics of human longevity.

Baggio G. Homocysteine and lipoprotein (a) levels in centenarians: a reappraisal of vascular risk factors.

Franceschi C. Centenarians: the genetic basis of successful ageing.

Herskind A.M. The Inheritance of human lifespan.

Jeune B. Centenarians: an historical perspective.

Mari D. Haemostasis factors and longevity. 